\title{
Nash consistent representation of effectivity functions through lottery models
}

Citation for published version (APA):

Peleg, B., \& Peters, H. J. M. (2005). Nash consistent representation of effectivity functions through lottery models. METEOR, Maastricht University School of Business and Economics. METEOR Research Memorandum No. 031 https://doi.org/10.26481/umamet.2005031

Document status and date:

Published: 01/01/2005

DOI:

10.26481/umamet.2005031

Document Version:

Publisher's PDF, also known as Version of record

\section{Please check the document version of this publication:}

- A submitted manuscript is the version of the article upon submission and before peer-review. There can be important differences between the submitted version and the official published version of record.

People interested in the research are advised to contact the author for the final version of the publication, or visit the DOI to the publisher's website.

- The final author version and the galley proof are versions of the publication after peer review.

- The final published version features the final layout of the paper including the volume, issue and page numbers.

Link to publication

\footnotetext{
General rights rights.

- You may freely distribute the URL identifying the publication in the public portal. please follow below link for the End User Agreement:

www.umlib.nl/taverne-license

Take down policy

If you believe that this document breaches copyright please contact us at:

repository@maastrichtuniversity.nl

providing details and we will investigate your claim.
}

Copyright and moral rights for the publications made accessible in the public portal are retained by the authors and/or other copyright owners and it is a condition of accessing publications that users recognise and abide by the legal requirements associated with these

- Users may download and print one copy of any publication from the public portal for the purpose of private study or research.

- You may not further distribute the material or use it for any profit-making activity or commercial gain

If the publication is distributed under the terms of Article $25 \mathrm{fa}$ of the Dutch Copyright Act, indicated by the "Taverne" license above, 


\title{
Nash consistent representation of effectivity functions through lottery models
}

\author{
Bezalel Peleg* Hans Peters ${ }^{\dagger}$
}

September 2005

\begin{abstract}
Effectivity functions for finitely many players and alternatives are considered. It is shown that every monotonic and superadditive effectivity function can be augmented with equal chance lotteries to a finite lottery model-i.e., an effectivity function that preserves the original effectivity in terms of supports of lotteries - which has a Nash consistent representation. In other words, there exists a finite game form which represents the lottery model and which has a Nash equilibrium for any profile of utility functions, where lotteries are evaluated by their expected utility. No additional condition on the original effectivity function is needed.
\end{abstract}

\section{Introduction}

For a group of individuals and a set of alternatives, an effectivity function (Moulin and Peleg, 1982) describes for each subgroup of individuals the subsets of alternatives for which the subgroup is "effective". What "effective"

*Department of Mathematics \& Center for Rationality and Interactive Decision Theory, The Hebrew University of Jerusalem, Feldman Building, Givat-Ram, 91904 Jerusalem, Israel. E-mail: pelegba@math.huji.ac.il. Financial support from the Research School METEOR of the University of Maastricht, and from the Dutch Science Foundation NWO (grant no. B 46-542) is gratefully acknowledged.

${ }^{\dagger}$ Department of Quantitative Economics, University of Maastricht, P.O. Box 616, 6200 MD Maastricht, The Netherlands. E-mail: h.peters@ke.unimaas.nl. 
means depends on the context. For instance, for an effectivity function derived from a game (form), to say that a subgroup of players is effective for a subset of outcomes means that these players can combine their strategies in such a way that the realized outcome of the game is an element of the particular subset. Another important usage of an effectivity function is to describe a constitution for a society (Gärdenfors, 1981; Peleg, 1998). Then, to say that a subgroup of individuals in the society is effective for a subset of alternatives (social states) means that this group is entitled to the realized social state being a state in that subset.

For this last application in particular, it is important to be able to "implement" the constitution by a set of rules (laws) according to which individuals should behave, such that their original rights as described by the constitution are preserved. In formal terms, it is important to find a game form - a set of rules or, more precisely, allowed actions and associated outcomes - of which the induced effectivity function is equal to the orginal effectivity function (the constitution). Additionally, it is important that such a game form possess some form of stability. A weak form of stability is the existence of a Nash equilibrium for every profile of individual preferences. In Peleg et al. (2002) a necessary and sufficient condition was found for an effectivity function to have a Nash consistent representation, i.e., a representing game form that has a Nash equilibrium for any profile of preferences. This result holds for a wide range of effectivity functions, including effectivity functions for finitely many individuals and alternatives, the case also studied in the present paper. The condition established in Peleg et al. (2002), however, is quite restrictive, and a natural question is whether it can be avoided by adapting the effectivity function or the representing game form. One way in which this could be done is to introduce mixed strategies in the game form and appeal to the existence of Nash equilibrium in mixed strategies. Since there are many reasons why the introduction of mixed strategy spaces in the model is not attractive, this is not the route that we follow.

Our approach to this problem is based on the concept of a lottery model for an effectivity function. This means that we add equal chance lotteries over alternatives in such a way that the original effectivity is preserved in the following sense. Suppose that a group of individuals $S$ is effective for a set of alternatives (say) $B$, then we assume that this effectiveness relation is preserved if $S$ is effective for a set of lotteries with total support equal to $B$. We further assume that lotteries are evaluated by individuals on the basis of their expected utilities (see Section 6 for more discussion on this 
issue and related literature). With these assumptions, we are able to prove that for any effectivity function (satisfying the usual necessary conditions of monotonicity and superaddivity) there exists a lottery model which has a Nash consistent representation, without imposing further conditions on the effectivity function. The representing game form is finite, and no mixed strategies are used.

For a constitution modelled as a monotonic and superadditive effectivity function, the relevance of this result is that such a constitution can always be "decentralized" by a set of rules (a game form) that preserves the original rights and that is stable in the sense that for any preferences a Nash equilibrium exists, as long as we are willing to accept some uncertainty in the form of equal chance lotteries as outcomes of the game, evaluated by expected utility.

For a given finite game form our result implies that we can always find an alternative finite game form, preserving effectivity in the indicated sense, that has a pure Nash equilibrium for any profile of preferences, again evaluating lotteries by their expected utilities. This also entails a solution to the socalled Gibbard paradox (Gibbard, 1974; see Example 4.3 in Section 4 below).

After preliminaries in Section 2, Section 3 extends the main result of Peleg et al. (2002) on Nash consistent representation of effectivity functions in order to be able to use restrictions in the domain of preferences - in our case to expected utility preferences. Section 4 introduces lottery models and presents the main result and some examples. In Section 5 we consider the case of neutral effectivity functions, for which a natural and simple lottery model can be based on the so-called uniform core. Section 6 concludes.

Notations For a finite set $D,|D|$ denotes the number of elements of $D$; $P(D)$ denotes the set of all subsets of $D ; P_{0}(D)$ denotes the set of all nonempty subsets of $D$.

\section{Effectivity functions and game forms}

Let $N=\{1, \ldots, n\}$ (where $n \geq 2$ ) be the set of players, and let $Z$ be a non-empty finite set. An effectivity function (EF) is a function $E: P(N) \rightarrow$ $P\left(P_{0}(Z)\right)$ that satisfies the following conditions: (i) $E(N)=P_{0}(Z)$; (ii) $E(\emptyset)=\emptyset$; (iii) $Z \in E(S)$ for every $S \in P_{0}(N)$. As a general interpretation, $X \in E(S)$ means that coalition $S$ can force the final "alternative" to be an 
element of $X$. The interpretation of the three conditions is fairly obvious.

An effectivity function $E$ is superadditive if it satisfies the following condition: If $S_{i} \in P_{0}(N)$ and $X_{i} \in E\left(S_{i}\right)$ for $i=1,2$, and $S_{1} \cap S_{2}=\emptyset$, then $X_{1} \cap X_{2} \in E\left(S_{1} \cup S_{2}\right)$. The effectivity function $E$ is monotonic if

$$
\left[X \in E(S), X^{*} \in P_{0}(Z), X \subseteq X^{*} \text {, and } S \subseteq S^{*}\right] \Rightarrow X^{*} \in E\left(S^{*}\right) .
$$

Observe that monotonicity means monotonicity with respect to the players as well as monotonicity with respect to the alternatives. Monotonicity and superadditivity of effectivity functions are natural properties in view of the interpretation given above. Moreover, effectivity functions derived from game forms (see below) have these properties.

Let $U$ be some non-empty set of utility functions $u: Z \rightarrow \mathbb{R}$. Then $U^{N}$ is the associated set of utility profiles. A function $u \in U$ expresses a preference that a player might have over the elements of $Z$.

We now turn to game forms. A game form $(\mathrm{GF})$ is an $(n+2)$-tuple $\Gamma=\left(\Sigma^{1}, \ldots, \Sigma^{n} ; \pi ; Z\right)$, where (i) $\Sigma^{i}$ is the (non-empty finite) set of strategies of player $i \in N$; and (ii) $\pi: \Sigma^{1} \times \cdots \times \Sigma^{n} \rightarrow Z$ is the outcome function. For $S \in P_{0}(N)$ we denote $\Sigma^{S}=\times_{i \in S} \Sigma^{i}$. Let $u^{N} \in U^{N}$. The pair $\left(\Gamma, u^{N}\right)$ defines, in an obvious way, a game in strategic form. A strategy combination $\sigma \in \Sigma^{N}$ is a Nash equilibrium of $\left(\Gamma, u^{N}\right)$ if

$$
\left[\tau^{i} \in \Sigma^{i}, i \in N\right] \Rightarrow u^{i}(\pi(\sigma)) \geq u^{i}\left(\pi\left(\sigma^{N \backslash\{i\}}, \tau^{i}\right)\right) .
$$

(Here $\sigma^{N \backslash\{i\}}$ is the restriction of $\sigma$ to $N \backslash\{i\}$.) We say that $\Gamma$ is Nash consistent on $U^{N}$ if $\left(\Gamma, u^{N}\right)$ has a Nash equilibrium for every $u^{N} \in U^{N}$.

Let $\Gamma=\left(\Sigma^{1}, \ldots, \Sigma^{n} ; \pi ; Z\right)$ be a game form and assume that $\pi$ is surjective. The effectivity function $E^{\Gamma}$, associated with $\Gamma$, is defined in the following way. For $S \in P_{0}(N)$ and $X \in P_{0}(Z), S$ is effective for $X$ if there exists $\sigma^{S} \in \Sigma^{S}$ such that $\pi\left(\sigma^{S}, \tau^{N \backslash S}\right) \in X$ for all $\tau^{N \backslash S} \in \Sigma^{N \backslash S}$. Then $E^{\Gamma}$ is defined ${ }^{1}$ by $E^{\Gamma}(\emptyset)=\emptyset$ and

$$
E^{\Gamma}(S)=\left\{X \in P_{0}(Z): S \text { is effective for } X\right\} \text {, for } S \in P_{0}(N) .
$$

Clearly, $E^{\Gamma}$ is superadditive and monotonic. Let $E: P(N) \rightarrow P\left(P_{0}(Z)\right)$ be an effectivity function. A game form $\Gamma$ is a representation of $E$ if $E(S)=$ $E^{\Gamma}(S)$ for every $S \in P_{0}(N)$. Basically, this means that the game form distributes the same power among the players as the effectivity function does.

\footnotetext{
${ }^{1} E^{\Gamma}$ is defined in Moulin and Peleg, 1982, where it is called the $\alpha$-EF of $\Gamma$.
} 


\section{Nash consistent representation of effectiv- ity functions}

In Peleg et al. (2002) a necessary and sufficient condition was derived for the existence of a Nash consistent representation of an effectivity function. Here, we give an extension of this result for an arbitrary set of utility functions.

Let $U$ be a non-empty set of utility functions $Z \rightarrow \mathbb{R}$, and let $X \in P_{0}(Z)$. Call $X$ admissible with respect to $U$ if there is a $u \in U$ such that $u(x)>u(y)$ for every $x \in X$ and every $y \in Z \backslash X$. Hence, a player with utility function $u$ strictly prefers every element of $X$ to every element not in $X$. For an effectivity function $E$ and for $i \in N$, let

$$
E^{*}(i):=\left\{X \in P_{0}(Z): X \cap Y \neq \emptyset \text { for all } Y \in E(N \backslash\{i\})\right\} .
$$

Theorem 3.1 Let $E: P(N) \rightarrow P\left(P_{0}(Z)\right)$ be a superadditive and monotonic effectivity function. Then $E$ has a representation that is Nash consistent on $U^{N}$ if and only if

$$
\left[X^{i} \in E^{*}(i) \text { and } X^{i} \text { admissible w.r.t. } U \text { for all } i \in N\right] \Rightarrow \bigcap_{i=1}^{n} X^{i} \neq \emptyset \text {. }
$$

A proof of this theorem is provided in the Appendix.

\section{$4 \quad$ Lottery models}

We will now be more specific about the set $Z$. Let $A$ be a finite set of (pure) alternatives, $|A| \geq 2$. For each $B \in P_{0}(A), \ell(B)$ denotes the lottery that assigns equal probability $1 /|B|$ to each alternative in $B$. The set of all such equal chance lotteries with support in a set $B \in P_{0}(A)$ is denoted by $\widetilde{B}$, hence

$$
\widetilde{B}=\left\{\ell\left(B^{\prime}\right): B^{\prime} \in P_{0}(B)\right\} .
$$

By identifying each $x \in A$ with the degenerate lottery $\ell(\{x\})$, we have $B \subseteq \widetilde{B}$.

Typically, we shall consider $Z=A$ and $Z=\widetilde{A}$. Let $\widetilde{E}: P(N) \rightarrow$ $P\left(P_{0}(\widetilde{A})\right)$ be an EF. With $\widetilde{E}$ we associate an EF $E: P(N) \rightarrow P\left(P_{0}(A)\right)$ as follows. Let $E(\emptyset)=\emptyset$. For $S \in P_{0}(N)$ and $B \in P_{0}(A)$, we let $B \in E(S)$ if there exists an $X \in \widetilde{E}(S)$ such that

$$
B=\bigcup_{B^{\prime} \in P_{0}(A): \ell\left(B^{\prime}\right) \in X} B^{\prime}
$$


In other words, elements of $E(S)$ are obtained by taking the union of the supports of elements of $\widetilde{E}(S)$. It is straightforward to check that, indeed, $E$ is an EF. If $E$ is derived from $\widetilde{E}$ in this way, then we call $\widetilde{E}$ a lottery model for $E$.

Remark 4.1 Monotonicity of $\widetilde{E}$ with respect to the players implies monotonicity of $E$ with respect to the players, and (since $A \subseteq \widetilde{A}$ ) monotonicity of $\widetilde{E}$ with respect to the alternatives implies monotonicity of $E$ with respect to the alternatives. Hence, monotonicity of $\widetilde{E}$ is inherited by $E$. Also superadditivity of $\widetilde{E}$ is inherited by $E$. For let $\widetilde{E}$ be superadditive, $S_{1}, S_{2} \in P_{0}(N)$ with $S_{1} \cap S_{2}=\emptyset$, and let $B_{1} \in E\left(S_{1}\right)$ and $B_{2} \in E\left(S_{2}\right)$. Let $X_{1} \in \widetilde{E}\left(S_{1}\right)$ and $X_{2} \in \widetilde{E}\left(S_{2}\right)$ correspond to $B_{1}$ and $B_{2}$ as in the definition of $E$, i.e., as in (2). Then superadditivity of $\widetilde{E}$ implies $X:=X_{1} \cap X_{2} \in \widetilde{E}\left(S_{1} \cup S_{2}\right)$, hence

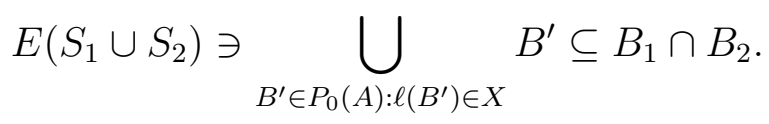

Monotonicity of $E$ now implies $B_{1} \cap B_{2} \in E\left(S_{1} \cup S_{2}\right)$. This shows that $E$ is superadditive.

The converse is not true: a lottery model $\widetilde{E}$ for a monotonic and superadditive $\mathrm{EF} E$ is not itself necessarily monotonic and superadditive.

Let $u: A \rightarrow \mathbb{R}$ be an arbitrary utility function, expressing preferences over pure alternatives. We assume that lotteries are evaluated by considering their expected utility value. Hence, we define

$$
V:=\left\{u \in \mathbb{R}^{\widetilde{A}}: u(\ell(B))=\sum_{a \in B} \frac{u(a)}{|B|} \text { for all } B \in P_{0}(A)\right\}
$$

to be the set of all expected utility functions on $\widetilde{A}$.

The main result of this paper is that for every monotonic and superadditive effectivity function there exists a lottery model which has a Nash consistent representation on $V^{N}$. Clearly, monotonicity and superadditivity cannot be left out here: a lottery model that has a representing game form must be monotonic and superadditive, and by Remark 4.1 the original "deterministic" EF must also be monotonic and superadditive. But, in contrast to Theorem 3.1, no additional condition is needed on $E$. 
Theorem 4.2 Let $E: P(N) \rightarrow P\left(P_{0}(A)\right)$ be a monotonic and superadditive $E F$. Then there exists an $E F \widetilde{E}: P(N) \rightarrow P\left(P_{0}(\widetilde{A})\right)$ such that

(i) $\widetilde{E}$ is a lottery model for $E$;

(ii) $\widetilde{E}$ has a representation which is Nash consistent on $V^{N}$.

Proof. Define $\widetilde{E}: P(N) \rightarrow P\left(P_{0}(\widetilde{A})\right)$ as follows. Let $\widetilde{E}(\emptyset)=\emptyset$ and for every $S \in P_{0}(N)$ with $|S| \neq n-1$ let

$$
\widetilde{E}(S)=\left\{X \in P_{0}(\widetilde{A}): X \supseteq \widetilde{B} \text { for some } B \in E(S)\right\} .
$$

Let $S \in P_{0}(N)$ with $|S|=n-1$, say $S=N \backslash\{i\}$ for some $i \in N$. For each $C \in E(N \backslash\{i\})$ and $C^{\prime} \in P_{0}(C)$ define the set $X\left(C, C^{\prime}\right) \in P_{0}(\widetilde{A})$ by

$$
X\left(C, C^{\prime}\right)=\left\{\begin{array}{cc}
\left\{\ell\left(\{c\} \cup C^{\prime}\right): c \in C \backslash C^{\prime}\right\} & \text { if } C^{\prime} \neq C \\
\{\ell(C)\} & \text { if } C^{\prime}=C .
\end{array}\right.
$$

Then let $X \in \widetilde{E}(N \backslash\{i\})$ if and only if $X \supseteq \widetilde{B}$ for some $B \in E(N \backslash\{i\})$ or $X \supseteq X\left(C, C^{\prime}\right) \cup \widetilde{B}$ for some $C \in E(N \backslash\{i\}), C^{\prime} \in P_{0}(C)$, and $B \in P_{0}(A)$ such that $B \cap B^{\prime} \neq \emptyset$ for all $B^{\prime} \in E(\{i\}$. This concludes the definition of $\widetilde{E}$. It is not hard to verify that $\widetilde{E}: P(N) \rightarrow P\left(P_{0}(\widetilde{A})\right)$ is a monotonic and superadditive EF and that $\widetilde{E}$ is a lottery model for $E$.

It remains to prove that $\widetilde{E}$ has a Nash consistent representation on $V^{N}$. For each $i \in N$, let $X^{i} \in \widetilde{E}^{*}(i)$ such that $X^{i}$ is admissible with respect to $V$. In view of Theorem 3.1 it is sufficient to prove $\bigcap_{i \in N} X^{i} \neq \emptyset$. For each $i \in N$ choose $u^{i} \in V$ such that

$$
u^{i}(x)>u^{i}(y) \text { for all } x \in X^{i} \text { and } y \in \widetilde{A} \backslash X^{i}
$$

(this is possible since each $X^{i}$ is admissible), and choose $B^{i} \in E(\{i\})$ and $b^{i} \in B^{i}$ such that

$$
u^{i}\left(b^{i}\right)=\min \left\{u^{i}(b): b \in B^{i}\right\} \geq \min \left\{u^{i}(b): b \in B\right\} \text { for all } B \in E(\{i\}) .
$$

Also, for each $i \in N$, define $C^{i}:=\bigcap_{j \in N \backslash\{i\}} B^{j}$. Then $C^{i} \in E(N \backslash\{i\})$ by superadditivity of $E$. Choose $a^{i} \in C^{i}$ such that

$$
u^{i}\left(a^{i}\right)=\max \left\{u^{i}(a): a \in C^{i}\right\} .
$$


By superadditivity of $E, u^{i}\left(a^{i}\right) \geq u^{i}\left(b^{i}\right)$. For every $i \in N$ define $\bar{B}^{i}:=$ $B^{i} \cup\left\{a^{i}\right\}$ and $\bar{C}^{i}:=\bigcap_{j \in N \backslash\{i\}} \bar{B}^{j}$. By monotonicity, still $\bar{B}^{i} \in E(\{i\})$ and $\bar{C}^{i} \in E(N \backslash\{i\})$ for every $i \in N$.

Now fix a player $i \in N$ and write $u^{i}\left(x_{1}\right) \geq u^{i}\left(x_{2}\right) \geq \ldots \geq u^{i}\left(x_{m}\right)$, where $A=\left\{x_{1}, x_{2}, \ldots, x_{m}\right\}$. Let $k \in\{1,2, \ldots, m\}$ such that $a^{i}=x_{k}$. Choose $p$ with $k \leq p \leq m$ such that

$$
u^{i}\left(\ell\left(\left\{x_{k}, x_{p}, x_{p+1}, \ldots, x_{m}\right\}\right)\right) \leq u^{i}\left(\ell\left(\left\{x_{k}, x_{p^{\prime}}, x_{p^{\prime}+1}, \ldots, x_{m}\right\}\right)\right)
$$

for all $k \leq p^{\prime} \leq m$. Consider the set $D=\left\{x_{k}, \ldots, x_{m}\right\}$. Since $\bar{C}^{i} \subseteq D$, we have $D \in E(N \backslash\{i\})$. Let $D^{\prime}:=\left\{x_{p}, x_{p+1}, \ldots, x_{m}\right\} \subseteq D$. Define $Y \in P_{0}(\widetilde{A})$ by

$$
Y=X\left(D, D^{\prime}\right) \cup F
$$

where

$$
F=\left\{b \in A: b \in B \text { with } u^{i}(b)=\min \left\{u^{i}(c): c \in B\right\} \text { for some } B \in E(i)\right\} .
$$

By definition of $\widetilde{E}$ and $Y$, we have $Y \in \widetilde{E}(N \backslash\{i\})$. It follows, in particular, that the set $X^{i}$ contains an element of $Y$, say $y$. Consider the lottery $\bar{\ell}=$ $\ell\left(\bigcap_{j \in N} \bar{B}^{j}\right)$. Then

$$
a^{i} \in \bar{B}^{i} \cap \bar{C}^{i}=\bigcap_{j \in N} \bar{B}^{j} \text { and } u^{i}\left(a^{i}\right) \geq u^{i}(c) \geq u^{i}\left(b^{i}\right) \text { for all } c \in \bigcap_{j \in N} \bar{B}^{j} .
$$

We show that $\bar{\ell} \in X^{i}$ by considering all the possible values for $y \in Y \cap X^{i}$. If $y \in F$, then $u^{i}(\bar{\ell}) \geq u^{i}\left(b^{i}\right) \geq u^{i}(y)$, where the first inequality follows from (4) and the last inequality by definition of $b^{i}$ and $F$. By (3), this implies $\bar{\ell} \in X^{i}$. If $y \in X\left(D, D^{\prime}\right)$, then $y=\ell\left(\left\{x_{p^{\prime}}, x_{p}, \ldots, x_{m}\right\}\right)$ for some $p^{\prime} \in\{k, k+1, \ldots, p-1\}$ if $k<m$ and $y=x_{m}$ if $k=m$. In that case, we argue as follows. Write $\bigcap_{j \in N} \bar{B}^{j}=\left\{a^{i}, y_{1}, \ldots, y_{r}\right\}$ with $u^{i}\left(a^{i}\right) \geq u^{i}\left(y_{1}\right) \geq$ $u^{i}\left(y_{2}\right) \geq \ldots \geq u^{i}\left(y_{r}\right)$. Then

$$
\begin{aligned}
u^{i}(\bar{\ell}) & \geq u^{i}\left(\ell\left(\left\{a^{i}, x_{m-r+1}, x_{m-r+2}, \ldots, x_{m}\right\}\right)\right) \\
& \geq u^{i}\left(\ell\left(\left\{a^{i}, x_{p}, \ldots, x_{m}\right\}\right)\right) \\
& \geq u^{i}\left(\ell\left(\left\{x_{p^{\prime}}, x_{p}, \ldots, x_{m}\right\}\right)\right) \\
& =u^{i}(y)
\end{aligned}
$$

where the second inequality follows from the choice of $p$, and the third follows since $u^{i}\left(a^{i}\right) \geq u^{i}\left(x_{p^{\prime}}\right)$. Hence also in this case, (3) implies that $\bar{\ell} \in X^{i}$. 
Since $i \in N$ was arbitrary, we conclude that $\bar{\ell} \in X^{j}$ for every $j \in N$, hence $\bigcap_{j \in N} X^{j} \neq \emptyset$.

A Nash consistent representation of the lottery model $\widetilde{E}$ in Theorem 4.2 is the one constructed in the proof of Theorem A.1 (see Peleg et al., 2002) in the Appendix, on which Theorem 3.1 is based. This particular game form has a number of appealing properties. In particular, it always admits a Pareto optimal Nash equilibrium outcome (cf. Remark A.2 in the Appendix). The following example illustrates the effectivity function $\widetilde{E}$, constructed in the proof of Theorem 4.2, for the effectivity function associated with a $2 \times 2$ game form.

Example 4.3 Let $N=\{1,2\}, A=\{a, b, c, d\}$, and consider the effectivity function $E$ derived from the game form

$$
\left[\begin{array}{ll}
a & b \\
c & d
\end{array}\right]
$$

where player 1 chooses a row and player 2 chooses a column. In particular, $E(\{1\})$ contains $\{a, b\},\{c, d\}$, and all supersets; $E(\{2\})$ contains $\{a, c\}$, $\{b, d\}$, and all supersets. It is easy to see that condition (1) in Theorem 3.1 is not fulfilled. For instance, $\{a, d\} \in E^{*}(\{1\}),\{b, c\} \in E^{*}(\{2\})$, both are admissible, but $\{a, d\} \cap\{b, c\}=\emptyset$.

The effectivity function $\widetilde{E}$, constructed in the proof of Theorem 4.2, assigns the following sets (where, e.g., $a b$ is shorthand for $\ell(\{a, b\})$, the equal chance lottery on $\{a, b\})$ :

$$
\begin{array}{rlrll}
\widetilde{E}(\{1\}): & \{a, b, a b\} & \{a, d, a b\} & \{c, b, a b\} & \{c, d, a b\} \\
& \{a, b, c d\} & \{a, d, c d\} & \{c, b, c d\} & \{c, d, c d\} \\
\widetilde{E}(\{2\}): & \{a, c, a c\} & \{a, d, a c\} & \{b, c, a c\} & \{b, d, a c\} \\
& \{a, c, b d\} & \{a, d, b d\} & \{b, c, b d\} & \{b, d, b d\}
\end{array}
$$

and all supersets of these sets within $\widetilde{A}$. It is easy to check that $\widetilde{E}$ is a lottery model for $E$. By the proof of Theorem 4.2, $\widetilde{E}$ has a Nash consistent representation. This can also be verified "directly" by using Theorem 3.1 and showing that $X^{1} \cap X^{2} \neq \emptyset$ for all admissible $X^{1} \in E^{*}(\{1\})$ and $X^{2} \in E^{*}(\{2\})$, but this is a rather tedious task. Admissibility has strong implications. For instance, if $a, b \in X^{1}$, then also $a b \in X^{1}$, or if $a b \in X^{1}$, then also $a \in X^{1}$ or $b \in X^{1}$, etc. 
The so called Gibbard paradox is formally an instance of this situation (see Gibbard, 1974; Gaertner et al., 1992; or Peleg et al., 2002). Our main result implies that the Gibbard paradox can be "solved" by allowing equal chance lotteries as described.

For particular cases, there may exist other lottery models that are less complex and in that sense more attractive. This is the case in the next example, where the unanimity effectivity function is considered.

Example 4.4 Let $E: P(N) \rightarrow P\left(P_{0}(A)\right)$ be the unanimity effectivity function, i.e., $E(S)=\{A\}$ for all $S \in P_{0}(N), S \neq N$. This EF clearly fails to satisfy condition (1). It is straightforward to check that here the lottery $\bar{\ell}$ in the proof of Theorem 4.2 is equal to $\ell(A)$, but in this case the lottery model $\widetilde{E}$ in that proof is overly complicated. It is again straightforward to see that also the effectivity function $\widetilde{E}^{\prime}$ is a lottery model for $E$, where for each $S \in P_{0}(N), S \neq N, \widetilde{E}^{\prime}(S)$ consists of $\{\ell(A)\}$ and all its supersets in $\widetilde{A}$, and $\widetilde{E}^{\prime}(N)=P_{0}(\widetilde{A})$. By applying Theorem 3.1 and checking condition (1) it follows that this lottery model has a Nash consistent representation.

Example 4.4 is a special case of a neutral effectivity function. These EFs are studied in the next section.

\section{$5 \quad$ Neutral effectivity functions}

A veto function is a function $v: P(N) \rightarrow\{-1,0, \ldots,|A|-1\}$ such that $v(\emptyset)=-1, v(S) \geq 0$ if $S \in P_{0}(N)$, and $v(N)=|A|-1$. The interpretation is that coalition $S$ can veto any subset of the alternatives with at most $v(S)$ elements. With $v$ we can associate a neutral (i.e., not depending on the names of the alternatives) effectivity function $E_{v}$ by

$$
E_{v}(S)=\left\{B \in P_{0}(A):|A \backslash B| \leq v(S)\right\}=\left\{B \in P_{0}(A):|B| \geq|A|-v(S)\right\}
$$

for every $S \in P(N)$. Conversely, it can be checked that every neutral effectivity function is derived from some veto function. A veto function is monotonic if $v(S) \leq v\left(S^{*}\right)$ for all $S, S^{*}$ with $S \subseteq S^{*}$, and superadditive if $v(S)+v\left(S^{*}\right) \leq v\left(S \cup S^{*}\right)$ for all $S, S^{*} \in P(N)$ with $S \cap S^{*}=\emptyset$. Clearly, a veto function is monotonic [superadditive] whenever the associated effectivity function is monotonic [superaditive]. 
We will show that for neutral effectivity functions there exists a simple and quite natural lottery model that has a Nash consistent representation. To this end we need the concept of the uniform core.

Let $E: P(N) \rightarrow P\left(P_{0}(A)\right)$ be a monotonic and superadditive effectivity function. Let $U=\mathbb{R}^{A}$ (in fact, it suffices to assume ordinal preferences here), $u^{N} \in U^{N}$, and say that $x \in A$ is uniformly dominated by $B \in P_{0}(A)$ via $S \in P_{0}(N)$ if (i) $B \in E(S)$; (ii) $x \notin B$; and (iii) $u^{i}(b)>u^{i}(a)$ for all $b \in B, a \in A \backslash B$, and $i \in S$. We also say that $S$ blocks $x$ by $B$. The set of all alternatives that are not uniformly dominated by some set $B$ via some coalition $S$ is called the uniform core and denoted $C_{\text {uniform }}\left(E, u^{N}\right)$. See Abdou and Keiding (1991), who show non-emptiness of the uniform core. The uniform core represents $E$ in the sense that, for every $S \in P_{0}(N)$ and every $B \in P_{0}(A)$,

$$
B \in E(S) \Leftrightarrow \exists u^{S} \in U^{S} \forall u^{N \backslash S} \in U^{N \backslash S}: C_{\text {uniform }}\left(E,\left(u^{S}, u^{N \backslash S}\right)\right) \subseteq B .
$$

See Keiding and Peleg (2004) for a proof of this fact.

Define $\widetilde{E}_{\text {uniform }}: P(N) \rightarrow P\left(P_{0}(\widetilde{A})\right)$ by requiring for each $S \in P_{0}(N)$ and $X \in P_{0}(\widetilde{A}):$

$$
X \in \widetilde{E}_{\text {uniform }}(S) \Leftrightarrow \exists u^{S} \in U^{S} \forall u^{N \backslash S} \in U^{N \backslash S}: \ell\left(C_{\text {uniform }}\left(E,\left(u^{S}, u^{N \backslash S}\right)\right)\right) \in X .
$$

Then $\widetilde{E}_{\text {uniform }}$ is a monotonic and superadditive lottery model for $E$. In fact, $\widetilde{E}_{\text {uniform }}=E^{\Gamma_{\text {uniform }}}$ where $\Gamma_{\text {uniform }}=(U, \ldots, U ; \pi ; \widetilde{A})$ is the game form defined by $\pi\left(u^{N}\right)=\ell\left(C_{\text {uniform }}\left(E, u^{N}\right)\right)$ for each $u^{N} \in U^{N}$. Hence, the game form $\Gamma_{\text {uniform }}$ represents the effectivity function $\widetilde{E}_{\text {uniform }}$, which in turn is a lottery model for $E$. We will show that $\Gamma_{\text {uniform }}$ is Nash consistent. Observe that $\Gamma_{\text {uniform }}$ is a simple game form, where each player just reports a utility function - in fact, it is sufficient to report a weak ordering over the alternatives. Given such a profile of reports one computes the uniform core and the outcome of the game is the equal chance lottery over the elements of the uniform core.

Theorem 5.1 Let $u^{N} \in U^{N}$. Then the game $\left(\Gamma_{\text {uniform }}, u^{N}\right)$ has a Nash equilibrium.

Proof. We construct a strategy profile $\hat{u}^{N} \in U^{N}$ inductively as follows. First, let $W(1) \subseteq A$ contain exactly $v(1)$ worst alternatives according to $u^{1}$, that is, $u^{1}(x) \leq u^{1}(y)$ for all $x \in W(1)$ and $y \in A \backslash W(1)$. Define $\hat{u}^{1}(x)=0$ 
and $\hat{u}^{1}(y)=1$ for all $x \in W(1)$ and $y \in A \backslash W(1)$. Let $k \in\{2, \ldots, n\}$ and suppose that $\hat{u}^{l}$ has been defined for all $1 \leq l \leq k-1$. Then let $W(k) \subseteq A$ contain exactly $v(k)$ worst alternatives in $A \backslash \bigcup_{l=1}^{k-1} W(l)$ acording to $u^{k}$, and define $\hat{u}^{k}(x)=0$ and $\hat{u}^{k}(y)=1$ for all $x \in W(k)$ and $y \in A \backslash W(k)$.

We claim that $\hat{u}^{N}$ is a Nash equilibrium in $\left(\Gamma_{\text {uniform }}, u^{N}\right)$. Let $k \in N$ and assume that each player $l \in N \backslash\{k\}$ plays the strategy $\hat{u}^{l}$. Consider any coalition $S \subseteq N \backslash\{k\}$ with more than one player. Then $S$ could only possibly block some alternative by the set $A \backslash W(l)$ for some $l \in S$, but all these sets are different since all sets $W(l)$ are different. Hence, only singletons in $N \backslash\{k\}$ block: each $l \in N \backslash\{k\}$ blocks $W(l)$, so altogether the set $\bigcup_{l \in N \backslash\{k\}} W(l)$ is blocked by the single players in $N \backslash\{k\}$. Consider the decision problem for player $k$. By the same argument as before, a non-singleton coalition $S$ containing player $k$ can only possibly block some alternative if $S=\{k, j\}$ for some $j \neq k$ (since all sets $W(l), l \in N \backslash\{k\}$ are different), but in that way $S$ can only block the set $W(j)$, namely by player $k$ playing some strategy $u^{\prime}$ such that $u^{\prime}(x)<u^{\prime}(y)$ for all $x \in W(j)$ and $y \in A \backslash W(j)$. Then the game would result in the equal chance lottery $\ell\left(A \backslash \bigcup_{l \in N \backslash\{k\}} W(l)\right)$. By only using his own blocking power, however, player $k$ can make sure that the outcome of the game is $\ell\left(A \backslash \bigcup_{l \in N} W(l)\right)$ by playing $\hat{u}^{k}$. Clearly, this is an improvement for player $k$, and also the best outcome attainable by using $k$ 's own blocking power.

Note that this proof depends on the assumption that indifferences between pure alternatives are allowed, in contrast to Theorem 4.2, which holds also if preferences between pure alternatives can only be strict.

The Nash equilibrium exhibited in the proof of Theorem 5.1 is a very natural one, since it consists of successive sincere vetoing of alternatives. Of course, vetoing according to any other ordering of the players would also be a Nash equilibrium.

If $E$ is non-neutral, then $\widetilde{E}_{\text {uniform }}$ is still a lottery model for $E$ and $\widetilde{E}_{\text {uniform }}=$ $E^{\Gamma_{\text {uniform }}}$, but it is not clear whether $\Gamma_{\text {uniform }}$ is still Nash consistent.

\section{Concluding remarks}

In this paper we have proved that every (monotonic and superadditive) effectivity function can be augmented, by adding finitely many equal chance lotteries, to a new effectivity function (lottery model) which preserves the 
original effectivity and has a Nash consistent representation. This approach is based on two critical assumptions.

First, we assume that in the lottery model the original effectiveness of a coalition $S$ of players for a set $B$ of alternatives is preserved if $S$ is now effective for some set $X$ of equal chance lotteries such that the union of the supports of the lotteries in $X$ is equal to $B$. For instance, if $B=\{a, b, c\}$, then $X$ could be the one-point set $\{\ell(\{a, b, c\})\}$ but also the two-point set $\{a, \ell(\{a, b, c\})\}$. This example shows that, in this set-up, we cannot really interpret effectiveness for $B$ as the alternatives of $B$ being equiprobable, even if we only add equal chance lotteries. Rather, players (or coalitions) evaluate effectiveness purely in terms of supports.

Second, we assume that equal chance lotteries resulting as outcomes of the representing game form are evaluated in terms of their expected utility. This assumption is not innocent since it is not obvious how expected utility maximization in general can be derived from underlying preferences if only this limited set of lotteries is available. This critical point, however, can be given a positive turn by observing that actually the expected utility property is only needed to evaluate equal chance lotteries, and may therefore be derived from a different set of assumptions in a decision model that only includes equal chance lotteries. An early example is Fishburn (1972), where preferences on sets of alternatives are considered and the expected utility property for equal chance lotteries is derived from conditions on these preferences. The assumption of equal chance lotteries evaluated by expected utility has been made frequently in the social choice literature, such as recently in Barberà et al. (2001), but also in earlier work, e.g., Feldman (1980). Indeed, also these references point at the interpretation of considering as outcomes sets rather than equal chance lotteries, and evaluating these sets using expected utility with equal chances. In fact, this was done in Section 5, where we considered the uniform core and evaluated that set as an equal chance lottery.

Finally, our result is also a contribution to the classical "purification" problem. For any finite game form, it enables us to construct a new finite game form which preserves the strategic possibilities of players and coalitions in the sense that the associated effectivity function is a lottery model for the effectivity function associated with the original game form, and which has a pure Nash equilibrium for any profile of utility functions respecting expected utility on equal chance lotteries. 


\section{A Appendix: Proof of Theorem 3.1}

For the proof of Theorem 3.1 we recall the following result, which is Theorem A.1 in Peleg et al. (2002). First a notation: for $u: Z \rightarrow \mathbb{R}$ and $x \in Z$, $L(x, u)=\{z \in Z: u(z) \leq u(x)\} .(L(x, u)$ is the lower contour set of $u$ at $x$.)

Theorem A.1 Let $E: P(N) \rightarrow P\left(P_{0}(Z)\right)$ be an effectivity function and let $U \subseteq \mathbb{R}^{Z}$. Then $E$ has a Nash consistent representation on $U^{N}$ if and only if the following conditions are satisfied:

(a) E is monotonic and superadditive.

(b) For every $u^{N} \in U^{N}$ there exists an $x \in Z$ such that $L\left(x, u^{i}\right) \in E(N \backslash\{i\})$ for all $i \in N$.

Remark A.2 The representing game form used in the proof of Theorem A.1 has two interesting properties. First, it is maximal in the sense that it admits any potential Nash equilibrium outcome, i.e., any outcome that is a Nash equilibrium (for a given utility profile) in some representing game form. Second, for any utility profile it admits a Pareto optimal Nash equilibrium outcome.

Proof of Theorem 3.1. First assume that $E$ has a Nash consistent representation $\Gamma$ on $U^{N}$. For each $i \in N$, let $X^{i} \in E^{*}(i)$ be an admissible set and $u^{i} \in U$ such that $u^{i}(z)>u^{i}(y)$ for all $z \in X^{i}$ and $y \in Z \backslash X^{i}$. Let $x \in Z$ be a Nash equilibrium outcome of $\left(\Gamma, u^{N}\right)$. Then

$$
L\left(x, u^{i}\right) \in E^{\Gamma}(N \backslash\{i\})=E(N \backslash\{i\}) \text { for all } i \in N .
$$

This implies $X^{i} \cap L\left(x, u^{i}\right) \neq \emptyset$ for every $i \in N$. By admissibility, $x \in X^{i}$ for all $u$, hence $\bigcap_{i \in N} X^{i} \neq \emptyset$, so that (1) holds.

For the converse, assume (1). Let $u^{N} \in U^{N}$. For every $i \in N$ let $Y^{i}:=$ $\left\{y \in Z: Z \backslash L\left(y, u^{i}\right) \in E^{*}(i)\right\}$ and define

$$
X^{i}=\left\{\begin{array}{cl}
\bigcap_{y \in Y^{i}} Z \backslash L\left(y, u^{i}\right) & \text { if } Y^{i} \neq \emptyset \\
Z & \text { otherwise. }
\end{array}\right.
$$

By $(1), \bigcap_{i \in N} X^{i} \neq \emptyset$. Take $x \in \bigcap_{i \in N} X^{i}$. Then $Z \backslash L\left(x, u^{i}\right) \notin E^{*}(i)$ for each $i$. Hence, there is some set $Z^{i} \subseteq L\left(x, u^{i}\right)$ with $Z^{i} \in E(N \backslash\{i\}$, so by monotonicity of $E, L\left(x, u^{i}\right) \in E(N \backslash\{i\})$ for every $i$. Theorem A.1 now implies that $E$ has a Nash consistent representation. 


\section{References}

Abdou, J., and H. Keiding (1991): Effectivity Functions in Social Choice. Dordrecht: Kluwer Academic Press.

Barberà, S., B. Dutta, and A. Sen (2001): "Strategy-Proof Social Choice Correspondences," Journal of Economic Theory, 101, 374-394.

Feldman, A.M. (1980): "Strongly Manipulable Multi-Valued Collective Choice Rules," Kyklos, International Review of Social Sciences, 33.

Fishburn, P.C. (1972): "Even-Chance Lotteries in Social Choice Theory," Theory and Decision, 3, 18-40.

Gaertner, W., P.K. Pattanaik, and K. Suzumura (1992): "Individual Rights Revisited," Economica, 59, 161-177.

Gärdenfors, P. (1981): "Rights, Games, and Social Choice," Noûs, 15, 341356 .

Gibbard, A. (1974): "A Pareto-Consistent Libertarian Claim," Journal of Economic Theory, 7, 388-410.

Keiding, H., and B. Peleg (2004): "Binary Effectivity Rules," The Hebrew University of Jerusalem.

Moulin, H., and B. Peleg (1982): "Cores of Effectivity Functions and Implementation Theory," Journal of Mathematical Economics, 10, 115-145.

Peleg, B. (1998): "Effectivity Functions, Game Forms, Games, and Rights," Social Choice and Welfare, 15, 67-80.

Peleg, B., H. Peters, and T. Storcken (2002): "Nash Consistent Representation of Effectivity Functions: A Reaction to the Gibbard Paradox," Mathematical Social Sciences, 43, 267-287. 